\title{
Age-related increase of oxidative stress-induced apoptosis in mice Prevention by Ginkgo biloba extract (EGb761)
}

\author{
K. Schindowski*, S. Leutner, S. Kressmann, A. Eckert, and W. E. Müller \\ Department of Pharmacology, Biocenter, Johann Wolfgang Goethe-University, \\ Frankfurt am Main, Federal Republic of Germany
}

Accepted April 18, 2001

\begin{abstract}
Summary. Enhanced apoptosis and elevated levels of reactive oxygen species (ROS) play a major role in aging. In addition, several neurodegenerative diseases are associated with increased oxidative stress and apoptosis in neuronal tissue. Antioxidative treatment has neuro-protective effects. The aim of the present study was to evaluate changes of susceptibility to apoptotic cell death by oxidative stress in aging and its inhibition by the antioxidant Ginkgo biloba extract EGb761. We investigated basal and ROS-induced levels of apoptotic lymphocytes derived from the spleen in young (3 months) and old (24 months) mice. ROS were induced by 2-deoxy-D-ribose (dRib) that depletes the intracellular pool of reduced glutathione. Lymphocytes from aged mice accumulate apoptotic cells to a significantly higher extent under basal conditions compared to cells from young mice. Treatment with dRib enhanced this difference, implicating a higher sensitivity to ROS in aging. Apoptosis can be reduced in vitro by treatment with EGb761. In addition, mice were treated daily with $100 \mathrm{mg} / \mathrm{kg}$ EGb761 per os over a period of two weeks. ROS-induced apoptosis was significantly reduced in the EGb761 group. Interestingly, this effect seemed to be more pronounced in old mice.
\end{abstract}

Keywords: Reactive oxygen species, aging, lymphocyte, programmed cell death, 2-deoxy-D-ribose.

\section{Introduction}

Under normal physiological conditions, oxygen consumption by cells with aerobic metabolism generates always potentially deleterious reactive oxygen species (ROS), which are usually detoxified by several enzymes and physiological antioxidants. A mismatch between production and detoxification of ROS leads to a state called oxidative stress and consequently to cellular

\footnotetext{
* Present address: Neuroanatomy, University of Heidelberg, Federal Republic of Germany
} 
damage. Oxidative stress seems to increase with age and is a major causal factor of cellular damage and enhanced apoptosis in many aging tissues including brain and the immune system (Buttke and Sandstrom, 1994; Leutner et al., 2001).

Apoptosis is of particular interest not only in aging but also in many degenerative diseases where it plays an important role in the pathological processes. In the immune system, apoptosis of white blood cells could be one reason for the immunosenescence related to cancer, infections and autoimmune disorders (Bender et al., 1986).

Recently, our group (Schindowski et al., 2000) showed that peripheral blood mononuclear cells (PBMC) of aged humans display a significantly higher content of basal apoptotic nuclei. In addition, oxidative stress-induced apoptosis was significantly higher in aged compared to young individuals. The reducing sugar 2-deoxy-D-ribose (dRib) induces oxidative stress in several kinds of cells (Barbieri et al., 1994; Ceruti et al., 1997) by depletion of intracellular glutathione (GSH), leading to apoptosis (Eckert et al., 1998a; Schindowski et al., 2000). Therefore, dRib provides a powerful tool to generate oxidative stress intracellularly and to induce apoptosis in vitro. In addition, we found that apoptosis of peripheral cells was increased in Alzheimer's disease (Schindowski et al., 1999). Most recently, we demonstrated that lymphocytes from presenilin-1 transgenic mice show a similar hypersensitivity to cell death as do peripheral cells from AD patients (Eckert et al., 2001b). These data further emphasize the excellent suitability of murine lymphocytes to study mechanisms of cell death and to monitor the protective efficacy of potential therapeutic drugs in preclinical studies. In order to demonstrate that enhanced apoptosis in aged humans is related to the aging process and not due to presymptomatic Alzheimer's disease, we investigated the basal and oxidative stress-induced apoptosis in lymphocytes from young and aged mice to exclude changes associated with AD. Moreover, the effects of the Ginkgo biloba extract EGb761 on murine lymphocyte apoptosis were studied in vitro and ex vivo in young and aged mice. The rationale for the pharmacological intervention study is as follows: EGb761 is a potent antioxidant and has already been shown to inhibit apoptosis in tissue culture experiments (Ni et al., 1996; Ahlemeyer et al., 1999; Bastianetto et al., 2000a) and oxidative stress in general (Kose et al., 1997; Pietri et al., 1997). Its effectiveness in Alzheimer's disease has been demonstrated by recent trials (Kanowski et al., 1996; Le Bars et al., 1997; Maurer et al., 1997; Oken et al., 1998).

\section{Methods}

\section{Animals}

Young ( 3 months) and old (24 months) female NMRI mice used in this study were from Harlan Winkelmann (Borchen, Germany). The latter were obtained at an age of 12 months and maintained at the Biocenter's animal care facility until use. All animals were housed in plastic cages with water and food ad libitum and were maintained on a 12-hour light/dark cycle. Mice with skin lesions, spleenomegaly and macroscopically visible tumors were excluded. For the ex vivo study, mice were treated daily with $100 \mathrm{mg} / \mathrm{kg}$ EGb761 per os in $0.2 \%$ agarose or with vehicle alone for 14 days. All experiments were performed in accordance with the German animal right regulations. 


\section{Reagents}

2-deoxy-D-ribose (dRib) and propidium iodide (PI) were purchased from Sigma (Taufkirchen, Germany). Anti-murine CD3-FITC-coupled monoclonal antibody was from PharMingen (Heidelberg, Germany) and 7-AAD from Molecular Probes (Leiden, The Netherlands). Anti-murine Pan-B beads were obtained from Dynal (Sweden). RPMI-1640, fetal calf serum (FCS), penicillin, streptomycin, phosphate buffered saline (PBS) and ethidium bromide stock solution were purchased from GibcoBRL LifeTechnologies (Karlsruhe, Germany). Proteinase K and RNase A were from Roche/ Boehringer Mannheim (Mannheim, Germany). All other mentioned chemicals were obtained from Merck (Darmstadt, Germany). Ginkgo biloba extract EGb761 was a kind gift of Schwabe GmbH (Karlsruhe, Germany).

\section{Cell preparation}

Mice were killed by cervical dislocation. T cells were isolated from the spleen with magnetic beads according to the manufacturer's instructions. In brief, spleen cell suspensions were prepared by mechanical dissociation of individual spleens in PBS supplemented with $10 \%$ FCS, penicillin $(100 \mathrm{U} / \mathrm{ml})$ and streptomycin $(100 \mu \mathrm{g} / \mathrm{ml})$. Red blood cells were lysed in hypotonic buffer $\left(155 \mathrm{mM} \mathrm{NH}_{4} \mathrm{Cl}\right.$ and $\left.10 \mathrm{mM} \mathrm{KHCO}_{3}\right)$ for 2 minutes. For B cell depletion, cells were washed twice, mixed with magnetic beads specific for $\mathrm{B}$ cells and incubated with shaking for 20 minutes at $4{ }^{\circ} \mathrm{C}$. T cell content of spleen cells was determined by $\mathrm{CD} 3$-staining to be over $80 \%$. For short-term culture, lymphocytes were seeded at $5 \times 10^{5}$ cells $/ \mathrm{ml}$ in complete medium (RPMI-1640 supplemented with $10 \%$ FCS, penicillin $100 \mathrm{U} / \mathrm{ml}$ and streptomycin $100 \mu \mathrm{g} / \mathrm{ml}$ ) at $37^{\circ} \mathrm{C}$ and $5 \% \mathrm{CO}_{2}$ in humidified atmosphere. Cells were cultured in the absence and presence of $10 \mathrm{mM}$ dRib for 24 hours. When indicated, spleen cells were preincubated with EGb761 for 1 hour before the addition of dRib.

\section{Detection of apoptosis with flow cytometry}

Cells were harvested at the indicated times by centrifugation $(200 \times \mathrm{g}, 5$ minutes $)$, and resuspended in $50 \mu \mathrm{g} / \mathrm{ml}$ propidium iodide in Na-citrate and $0.1 \%$ Triton X-100 for at least 10 minutes at $4{ }^{\circ} \mathrm{C}$ in the dark (Nicoletti et al., 1991). DNA fragmentation was analyzed by flow cytometry with FACSCalibur equipment using Cell Quest Software (Becton Dickinson, Heidelberg, Germany) as previously described (Schindowski et al., 2000). Sub- $\mathrm{G}_{0}$ DNA content is defined as percentage of apoptotic cells.

For all conditions investigated, necrosis was excluded in preceding experiments by staining with 7-Aminoactinomycin (7-AAD) according to the manufacturer's instruction.

\section{DNA ladder}

Cells were harvested by centrifugation $(200 \times$ g, 5 minutes $)$ and washed with PBS. Cell pellets were incubated in $50 \mathrm{mM}$ TRIS ( $\mathrm{pH} 7.5$ ) containing $100 \mathrm{mM} \mathrm{NaCl}, 1 \mathrm{mM}$ EDTA, $1 \%$ SDS and $20 \mu \mathrm{g} / \mathrm{ml}$ proteinase $\mathrm{K}$ at $55^{\circ} \mathrm{C}$ overnight. After digestion with RNase A, nucleic acids were extracted with phenol/chloroform and precipitated with $70 \%$ ethanol according standard procedures. Genomic DNA was electrophoresed in a 1\% agarose gel and stained with ethidium bromide.

\section{Statistics}

Statistical significance was determined by Student's t-test with Graph Pad Software Prism 3.0. Data presented are means \pm S.E.M. Results were considered to be statistically significant when $\mathrm{p}<0.05$. 


\section{Results}

\section{Basal levels of apoptotic nuclei}

$\mathrm{T}$ cells were isolated from the spleens of young ( 3 months) and old (24 months) mice. To reduce activation or cell death of T cells during the isolation procedure, B lymphocytes were removed by magnetic beads in order to leave the T cells untouched. Basal DNA fragmentation assessed immediately after isolation of the cells was analyzed by propidium iodide (PI) staining. Fluorescence intensity was plotted against number of counts. Significantly increased basal apoptotic levels could be determined in freshly isolated spleen cells from old mice $(2.18 \pm 0.23 \%$ apoptotic cells; $\mathrm{n}=13)$ compared to young mice $(1.77 \pm 0.26 \% ; \mathrm{n}=9 ; * \mathrm{p}<0.05 ;$ Fig. 1A).

\section{Oxidative stress enhances the age-related differences in apoptotic cell death}

2-deoxy-D-Ribose (dRib) depletes the intracellular pool of reduced glutathione thereby promoting states of enhanced oxidative stress inside the cell (Ceruti et al., 1997; Barbieri et al., 1994). In earlier studies we showed that oxidative stress induced by dRib significantly amplifies apoptotic cell death in human aging and in Alzheimer's disease (Schindowski et al., 2000). Here we demonstrate that dRib is able to induce apoptosis as well in murine lymphocytes (Fig. 1B). To further confirm the apoptotic nature of cell death, DNA laddering of nuclear fragmentation was assessed in murine $\mathrm{T}$ cells from individual mice. Figure 2 shows a representative experiment.

T cells of the spleen from young and old mice were incubated for 24 hours in the presence of $10 \mathrm{mM}$ dRib. In vitro apoptosis of cells incubated over the same period with medium alone was subtracted. Induction of oxidative stress revealed age-related changes in programmed cell death. A significant increase towards higher apoptotic levels was found in spleen cells of aged animals (old: $7.90 \pm 1.55 \%$; young: $2.21 \pm 0.45 \% ; * * p<0.01 ; \mathrm{n}=9-13$ in each group; Fig. 1B).

\section{In vitro effects of EGb761 on ROS-induced apoptosis in spleen cells from young and old mice}

To determine the protective potential of Ginkgo biloba extract on ROSinduced apoptosis in vitro, freshly isolated spleen cells from young and old mice were co-incubated with various doses of EGb761 and $10 \mathrm{mM} \mathrm{dRib}$ for 24 hours. The level of ROS-induced apoptosis (dRib alone) occurring after 24 hours of any individual animal was taken as $100 \%$ (full apoptotic effect). The percentage of basal apoptosis from freshly isolated lymphocytes was set as $0 \%$ (Fig. 3). Ginkgo biloba extract decreased dRib-induced apoptosis at concentrations above $10 \mu \mathrm{g} / \mathrm{ml}$. Interestingly, EGb761 provides greater protective properties in cells from aged animals. $250 \mu \mathrm{g} / \mathrm{ml}$ and $500 \mu \mathrm{g} / \mathrm{ml}$ revealed a significant reduction of apoptosis in aged mice compared to young mice. Since absolute levels of dRib-induced apoptosis are higher in lymphocytes from old animals (Fig. 1B), these findings demonstrate that cells from old mice specifically benefit from EGb761 treatment. In lymphocytes from young mice 


\section{A) basal levels of apoptosis}

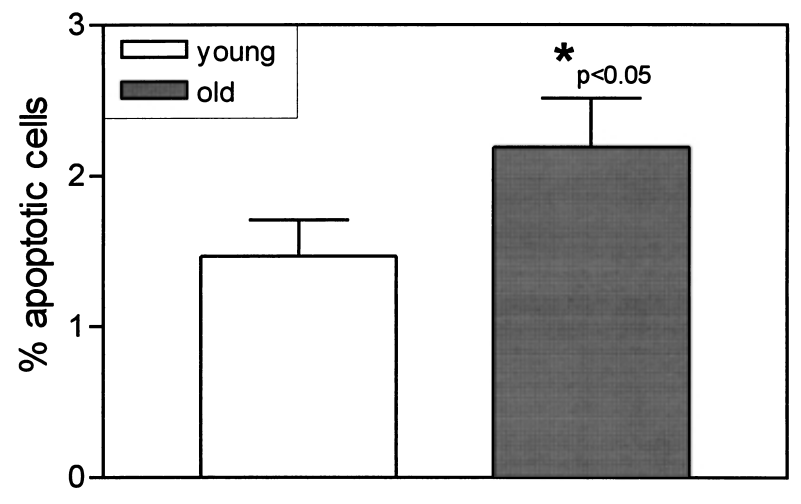

B) oxidative stress-induced apoptosis

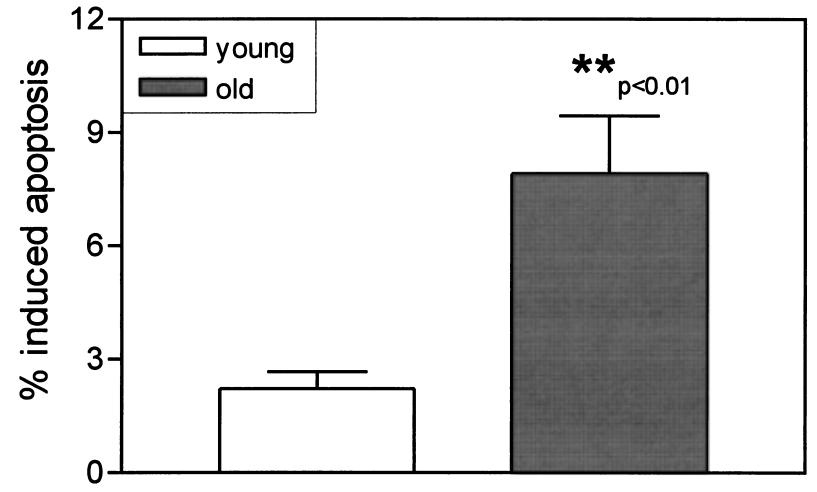

Fig. 1. Basal (A) and dRib-induced (B) apoptosis in T cells of young and old mice. Percent sub- $\mathrm{G}_{0}$ DNA content (termed as apoptotic cells) in freshly isolated lymphocytes derived from young ( 3 months of age, white bars) and old (24 months of age, gray bars) animals are shown. A Apoptotic cell death was determined immediately after isolation, B cells were incubated for 24 hours at $37^{\circ} \mathrm{C}$ in the presence of dRib $(10 \mathrm{mM})$. Data are means \pm S.E.M.; $\mathrm{n}=9-13$ per group, ${ }^{*} \mathrm{p}<0.05 ; * \mathrm{*} p<0.01$. Basal apoptosis describes the total amount of apoptotic cells of freshly isolated spleen cells. ROS-induced apoptosis is defined as the percentage of apoptosis in dRib-treated cultures subtracted by the spontaneous apoptosis, which occurs when culturing primary cells over a while under in vitro conditions. The spontaneous apoptosis was not altered between the two age groups

the reduction was less pronounced than in elderly animals. Two-way ANOVA revealed significant differences between mice groups and treatment with EGb761 (10-500 $\mu \mathrm{g} / \mathrm{ml} ; \mathrm{p}<0.05)$.

\section{Ex vivo effects of EGb761 on ROS-induced apoptosis in young and old mice}

In order to explore the effect of Ginkgo biloba extract on chronically treated cells and to determine its ex vivo anti-apoptotic function, we treated young and old mice with EGb761 in $0.2 \%$ agarose or with agarose alone as placebo over a period of 2 weeks. The dose used $(100 \mathrm{mg} / \mathrm{kg})$ has previously been 


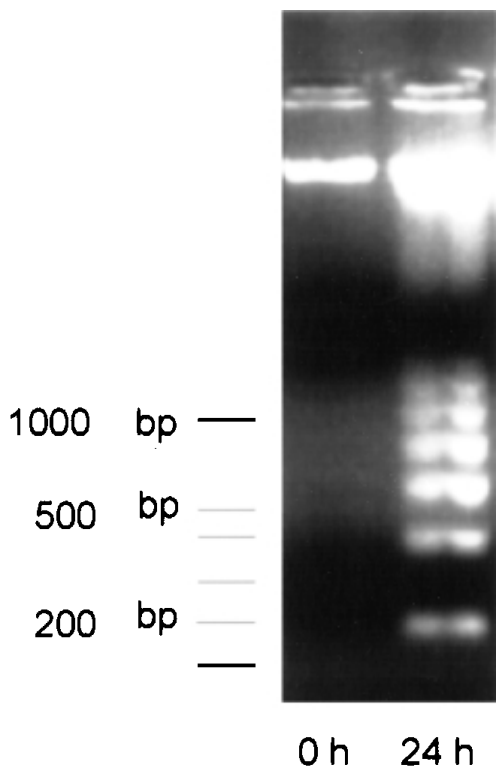

Fig. 2. Apoptotic cell death pattern in murine lymphocytes under basal conditions. Immediately after isolation, levels of apoptotic cells are too low, to give a detectable DNA ladder formation. After 24 hours incubation with dRib, a substantial apoptotic endonuclease activity occurs, which is evident by cleavage of chromosomal DNA between the nucleosomes producing oligomers of 180 basepairs (typical "DNA ladder")

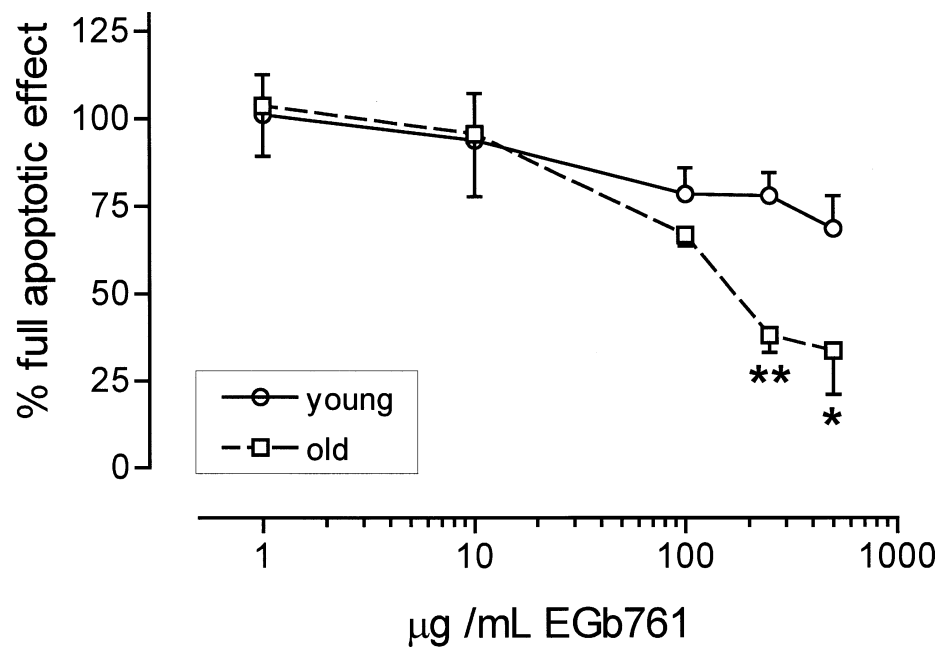

Fig. 3. Prevention of ROS-induced apoptosis by increasing doses of EGb761 in vitro. Percentage of apoptotic lymphocytes after 24 hours incubation without EGb761 treatment were defined as $100 \%$. Basal apoptosis of freshly isolated spleen cells was defined as $0 \%$. The anti-apoptotic effects of EGb761 at a concentration of $250 \mu \mathrm{g} / \mathrm{ml}$ and higher differ significantly between the two groups $(\mathrm{n}=3$ per group, $* * \mathrm{p}<0.01 ; * \mathrm{p}<0.05)$ 


\section{$\begin{array}{ll}\text { A) young } & \text { B) old }\end{array}$}

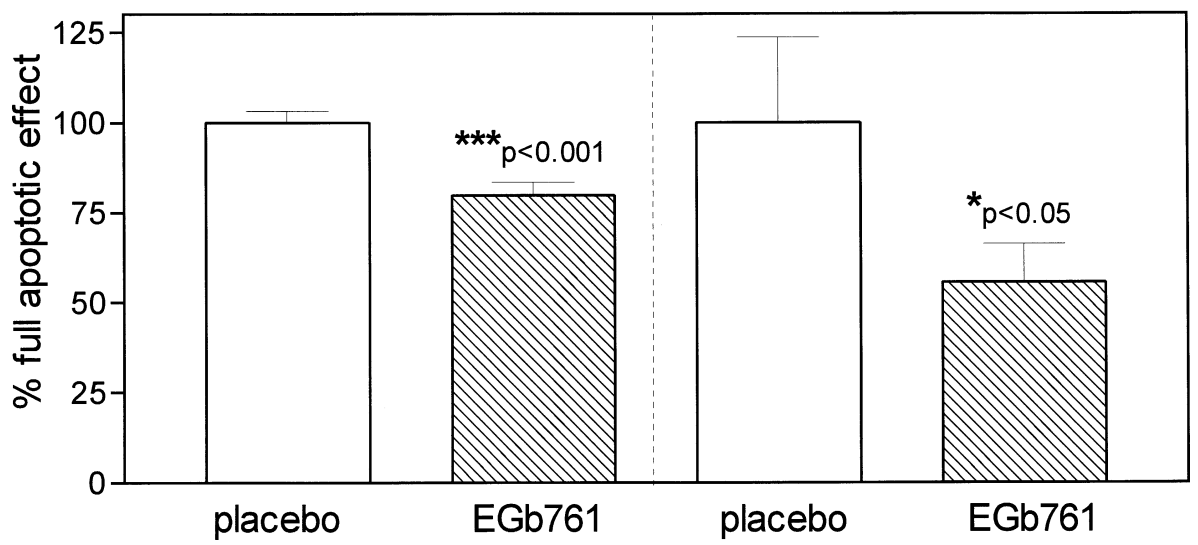

Fig. 4. Effect of oral EGb761-treatment on ROS-induced apoptosis in spleenic T cells from young and old mice. Young $(\mathbf{A})$ and old $(\mathbf{B})$ female NMRI mice were treated with $100 \mathrm{mg} / \mathrm{kg} \mathrm{EGb761} \mathrm{or} \mathrm{vehicle} \mathrm{orally} \mathrm{for} 2$ weeks. T cells were isolated from the spleens and incubated with $10 \mathrm{mM}$ dRib for 24 hours. Mean levels of dRib-induced apoptosis of the placebo group were set $100 \%$ (100\% full apoptotic effect). Basal apoptosis of freshly isolated spleen cells was defined as $0 \%$. A In spleen cells from young animals, ROSinduced apoptosis was diminished by EGb761 by about $20 \%$ when compared with placebo treated mice $(\mathrm{n}=9$ per group, $* * * \mathrm{p}<0.001)$. B In cells derived from old mice this effect was even more amplified. Apoptosis was reduced by about $45 \%$ in the EGb761treated group, though levels fluctuated more in aged animals $(\mathrm{n}=13$ per group, $* p<0.05)$. However, cells from aged mice revealed a significant greater benefit from EGb761-treatment than lymphocytes of young animals did [placebo old (B) vs. placebo young $\left.(\mathbf{A}):{ }^{\dagger} \mathrm{p}<0.05\right]$

shown to improve learning deficits in aged mice (Stoll et al., 1996). After 14 days of treatment, mice were killed by cervical dislocation and spleens were prepared as described above. No effect of EGb761 on basal apoptosis could be seen in either group (young: placebo; $1.77 \pm 0.26 \%$; EGb761, $1.76 \pm$ $0.28 \%, \mathrm{n}=9$ per group; old: placebo, $2.18 \pm 0.23 \%$; EGb761, $2.19 \pm 0.31 \%$, $\mathrm{n}=13$ per group), indicating no direct in vivo effect of EGb761 on physiological apoptosis. In contrast, ex vivo ROS-induced apoptosis triggered by treatment with dRib was significantly reduced in the placebo group of young animals (placebo: $100.0 \pm 3.10 \%$; EGb761: $79.80 \pm 3.58 \% ; n=9$ in each group; $* * * p<0.001$; Fig. 4A). Interestingly, as already shown by our in vitro experiments, lymphocytes from old animals revealed a significantly (placebo old: $55.55 \pm 10.65 \%$ vs. placebo young: $79.80 \pm 3.58 \%$; $*<<0.05)$ greater protection by the plant extract than did cells from younger mice (old, placebo: $100.0 \pm 23.51 \%$; EGb761 group $55.55 \pm 10.65 \% ; n=13$ per group; ${ }^{\dagger} \mathrm{p}<0.05$; Fig. 4B).

\section{Discussion}

ROS occur as physiological intermediates in aerobic metabolism and are usually eliminated inside cells by antioxidant pathways that require reduced 
glutathione (GSH) for regeneration. dRib provokes oxidative stress in cells. There are growing evidences that the underlying mechanism is diminishing the intracellular levels of GSH (Kletsas et al., 1998) and it seems to be therefore involved in the formation of free radicals (Ceruti et al., 1997; Schindowski et al., 2000). Depletion of GSH makes the cell defenseless against elevated levels of ROS, which can trigger the cell to undergo apoptosis by DNA damage or directly act as second messengers in the apoptotic pathway. Buttke and Sandstrom (1994) found that apoptosis in lymphocytes is often mediated by ROS, considering that already low concentrations of peroxides can initiate apoptosis. Therefore, dRib represents a useful tool to generate intracellular oxidative stress in lymphocytes (Barbieri et al., 1994; Eckert et al., 1998, 1998a). In recent studies, we clearly demonstrated that T-cells can cope less effectively with oxidative stress during aging in man and that this effect is amplified in Alzheimer's disease (Eckert et al., 1998b; Schindowski et al., 1999). In the present study, we confirmed that lymphocytes derived from old mice are also more vulnerable to dRib-induced apoptosis than lymphocytes from young mice. Proposing that dRib exhausts the glutathione pool, cells that generate more ROS should be more prone to apoptosis. Since apoptosis is significantly elevated in dRib treated cells from elderly mice, one can conclude that lymphocytes derived from old animals as compared with young, either generate more ROS during metabolism or are more sensitive to ROS. However, we can not exclude higher sensitivity of old animals to dRib.

Several in vitro studies using primary neurons in tissue culture have already shown that EGb761 can protect from oxidative stress-induced cell death (Bastianetto et al., 2000b; Chen et al., 1999; Ahlemeyer et al., 1999; Ni et al., 1996). Our results confirm and extend these findings by demonstrating that the protective effect of EGb761 is (1) also seen in fully differentiated lymphocytes in vitro and (2) can also be observed after ex vivo treatment where it seems to take place at lower concentrations. Moreover, our experiments reveal that EGb761 treatment has a higher protective effect in cells derived from elderly animals and that the degree of protection is similar in vitro and in vivo. In an earlier study, we demonstrated that dRib enhances significantly the levels of ROS in peripheral lymphocytes isolated from aged humans (Schindowski et al., 2000). Therefore, one might speculate that the percentage of prevention by EGb761 is greater, as more radicals generated by dRib can be eliminated. Even more important, apoptotic mechanisms needed for the physiological elimination of cells (basal apoptosis) were not affected by the EGb761 treatment. This fact may be relevant to the physiological role of programmed cell death in carcinogenesis, development, and homeostasis of the immune system. However, "pathological apoptosis" e.g. apoptosis induced by an additional stimulus, which seems to mimic enhanced oxidative stress in pathological conditions like Alzheimer disease, can be significantly prevented by Ginkgo treatment. Thus, the findings not only demonstrate that enhanced vulnerability to oxidative stress-induced apoptosis is a common feature of aged lymphocytes, but also that EGb761 might specifically interfere 
with this pathological mechanism, leaving physiological aspects of programmed cell death rather unaffected.

Based on our findings, lymphocytes seem to represent a suitable model system for monitoring drug effects on cell death mechanisms in further preclinical and clinical studies using EGb761 or other antioxidant drugs.

\section{Acknowledgements}

This study was supported from Schwabe Arzneimittel (Karlsruhe, Germany). We thank Dr. B. Steiner for her excellent help and critical comments on this manuscript.

\section{References}

Ahlemeyer B, Mowes A, Krieglstein J (1999) Inhibition of serum deprivation- and staurosporine-induced neuronal apoptosis by Ginkgo biloba extract and some of its constituents. Eur J Pharmacol 367: 423-430

Barbieri D, Grassilli E, Monti D, Salvioli S, Franceschini MG, Franchini A, Bellesia E, Salomoni P, Negro P, Capri M (1994) D-ribose and deoxy-D-ribose induce apoptosis in human quiescent peripheral blood mononuclear cells. Biochem Biophys Res Commun 201: 1109-1116

Bastianetto S, Ramassamy C, Dore S, Christen Y, Poirier J, Quirion R (2000a) The Ginkgo biloba extract (EGb 761) protects hippocampal neurons against cell death induced by beta-amyloid. Eur J Neurosci 12: 1882-1890

Bastianetto S, Zheng WH, Quirion R (2000b) The Ginkgo biloba extract (EGb 761) protects and rescues hippocampal cells against nitric oxide-induced toxicity: involvement of its flavonoid constituents and protein kinase C. J Neurochem 74: 2268-2277

Bender BS, Nagel JE, Adler WH, Andres R (1986) Absolute peripheral blood lymphocyte count and subsequent mortality of elderly men. The Baltimore Longitudinal Study of Aging. J Am Geriatr Soc 34: 649-654

Buttke TM, Sandstrom PA (1994) Oxidative stress as a mediator of apoptosis. Immunol Today 15: 7-10

Ceruti S, Barbieri D, Veronese E, Cattabeni F, Cossarizza A, Giammarioli AM, Malorni W, Franceschi C, Abbracchio MP (1997) Different pathways of apoptosis revealed by 2-chloro-adenosine and deoxy-D-ribose in mammalian astroglial cells. J Neurosci Res 47: 372-383

Chen C, Wei T, Gao Z, Zhao B, Hou J, Xu H, Xin W, Packer L (1999) Different effects of the constituents of EGb761 on apoptosis in rat cerebellar granule cells induced by hydroxyl radicals. Biochem Mol Biol Int 47: 397-405

Eckert A, Cotman CW, Zerfass R, Hennerici M, Müller WE (1998a) Enhanced vulnerability to apoptotic cell death in sporadic Alzheimer's disease. Neuroreport 9: 24432446

Eckert A, Cotman CW, Zerfass R, Hennerici M, Müller WE (1998b) Lymphocytes as cell model to study apoptosis in Alzheimer's disease: vulnerability to programmed cell death appears to be altered. J Neural Transm [Suppl] 54: 259-267

Eckert A, Oster M, Zerfass R, Hennerici M, Müller WE (2001a) Elevated levels of fragmented DNA nucleosomes in native and activated lymphocytes indicate an enhanced sensitivity to apoptosis in sporadic Alzheimer's disease. Dement Geriatr Cogn Disord 12: 98-105

Eckert A, Schindowski K, Leutner S, Luckhaus C, Touchet N, Czech C, Müller WE (2001b) Alzheimer's disease-related alterations in peripheral cells from presenilin-1 transgenic mice. Neurobiol Dis 8: 331-342

Kanowski S, Herrmann WM, Stephan K, Wierich W, Horr R (1996) Proof of efficacy of the ginkgo biloba special extract EGb 761 in outpatients suffering from mild to 
moderate primary degenerative dementia of the Alzheimer type or multi-infarct dementia. Pharmacopsychiatry 29: 47-56

Kletsas D, Barbieri D, Stathakos D, Botti B, Bergamini S, Tomasi A, Monti D, Malorni W, Franceschi C (1998) The highly reducing sugar 2-deoxy-D-ribose induces apoptosis in human fibroblasts by reduced glutathione depletion and cytoskeletal disruption. Biochem Biophys Res Commun 243: 416-425

Kose K, Dogan P, Ascioglu M, Ascioglu O (1997) In vitro antioxidant effect of Ginkgo biloba extract (EGb761) on lipoperoxidation induced hydrogen peroxide in erythrocytes of Behcet's patients. Jpn J Pharmacol 75: 253-258

Le Bars PL, Katz MM, Berman N, Itil TM, Freedman AM, Schatzberg AF (1997) A placebo-controlled, double-blind, randomized trial of an extract of Ginkgo biloba for dementia. North American EGb Study Group. JAMA 278: 1327-1332

Leutner S, Eckert A, Müller WE (2001) ROS generation, lipid peroxidation and antioxidant enzyme activities in the aging brain. J Neural Transm (this volume)

Maurer K, Ihl R, Dierks T, Fröhlich L (1997) Clinical efficacy of Ginkgo biloba special extract EGb761 in dementia of the Alzheimer type. J Psychiatr Res 31: 645-655

Ni Y, Zhao B, Hou J, Xin W (1996) Preventive effect of Ginkgo biloba extract on apoptosis in rat cerebellar neuronal cells induced by hydroxyl radicals. Neurosci Lett 214: $115-118$

Nicoletti I, Migliorati G, Pagliacci MC, Grignani F, Riccardi C (1991) A rapid and simple method for measuring thymocyte apoptosis by propidium iodide staining and flow cytometry. J Immunol Methods 139: 271-279

Oken BS, Storzbach DM, Kaye JA (1998) The efficacy of Ginkgo biloba on cognitive function in Alzheimer's disease. Arch Neurol 55: 1409-1415

Pietri S, Seguin JR, d'Arbigny P, Drieu K, Culasci M (1997) Ginkgo biloba extract (EGb761) pretreatments limits free radical-induced oxidative stress in patients undergoing coronary bypass surgery. Cardiovasc Drugs Ther 11: 121-131

Schindowski K, Leutner S, Gorriz C, Fröhlich L, Maurer K, Eckert A, Müller WE (1999) Altered apoptosis in lymphocyte from Alzheimer's disease patients is not directly linked to changes in lymphocyte's subsets. Soc Neurosci 25: 2124

Schindowski K, Leutner S, Muller WE, Eckert A (2000) Age-related changes of apoptotic cell death in human lymphocytes. Neurobiol Aging 21: 661-670

Stoll S, Scheuer K, Pohl O, Muller WE (1996) Ginkgo biloba extract (EGb 761) independently improves changes in passive avoidance learning and brain membrane fluidity in the aging mouse. Pharmacopsychiatry 29: 144-149

Warner HR (1999) Apoptosis: a two-edged sword in aging. Ann NY Acad Sci 887: 1-11

Authors' address: Dr. K. Schindowski, Department of Pharmacology, Biocenter, Johann-Wolfgang Goethe University, Building N260, Marie-Curie-Strasse 9, D-60439 Frankfurt am Main, Federal Republic of Germany, e-mail: Schindowski@em.unifrankfurt.de. Present address: Neúroanatomy University of Heidelberg im Neúeuheimer Feld 307 D-69120 Heidelberg Federal Repúblic of Germany 